\title{
DYNAMICS OF THE LUNAR SPIN AXIS
}

\author{
JACK WISDOM \\ Massachusetts Institute of Technology, Cambridge, MA 02139; wisdom@mit.edu \\ Received 2005 June 29; accepted 2005 November 11
}

\begin{abstract}
The evolution of the lunar spin axis is studied. Prior work has assumed that the inclination of the lunar orbit is constant and that the node regresses uniformly. This work takes into account the nonconstant inclination and nonuniform regression of the node as determined from averaged models of the motion of the lunar orbit. The resulting dynamics is considerably more rich, exhibiting additional resonances, period doubling and tripling, and chaos.
\end{abstract}

Key words: celestial mechanics — gravitation — methods: analytical — methods: numerical — planets and satellites: formation — planets and satellites: individual (Earth, Moon)

\section{INTRODUCTION}

The spin axis of the Moon is very nearly in the same plane as the normal to the orbit of the Moon about the Earth and the normal to the Earth's orbit about the Sun. The spin axis and lunar orbit normal regress at the same rate about the normal to the Earth's orbit. This configuration is known as a Cassini state (Colombo 1966; Peale 1969). Peale (1969) studied the dynamics of Cassini states in the approximation that the orbit has a constant inclination and uniform regression and the spin is uniform and synchronous, as for the Moon, or commensurate with the orbital motion, as for Mercury. For this case there are, depending on parameters, two or four such dynamical equilibria in which the spin axis co-regresses with the orbit.

Ward (1975) and Peale \& Cassen (1978) studied the past orientation of the lunar spin axis and calculated the rate of tidal heating that results from the forced Cassini obliquity. At each epoch, the lunar orbit was assumed to have a given inclination and uniform regression, so that the simple theory of equilibrium Cassini states could be applied. Approximate values for the inclination and nodal regression rate were taken from the lunar tidal evolution model of Goldreich (1966). The picture that was developed is as follows. Early in the evolution of the Moon there were four Cassini states, and the Moon occupied the one with low obliquity, with prograde synchronous spin. The Moon evolved outward due to tidal friction, and at about $34 R_{\oplus}\left(R_{\oplus}\right.$ is the radius of the Earth) the stable equilibrium that the Moon occupied merged with the unstable equilibrium, leaving only two equilibrium Cassini states. The Moon went through a period of nonequilibrium oscillation, and, through internal friction, the spin eventually settled on the sole remaining Cassini state with low obliquity. As the Moon continued to evolve outward to the present configuration, the Moon remaind in this Cassini state.

Touma \& Wisdom (1993) and Laskar \& Robutel (1993) discovered that the obliquity of Mars evolves chaotically. Touma $\&$ Wisdom (1993) interpreted this result in terms of generalized Cassini dynamics in which the precession of Mars is nearly resonant with the second fundamental inclination mode of the solar system. The solar system evolves chaotically (Laskar 1989; Sussman \& Wisdom 1992), and the amplitude and frequency of this mode vary with time; in phase space the resonance slowly pulsates. Mars, approximately conserving an adiabatic invariant, is forced in and out of the resonance, and adiabatic chaos ensues (Wisdom 1985). Recently, it has been argued that Saturn is in a similar secular Cassini state (Ward \& Hamilton 2004).

The motion of the lunar orbit is complex; it does not regress uniformly with constant inclination to any plane. In this paper I reexamine the dynamics of the lunar spin axis throughout its history, taking into account the nonuniform regression of the lunar orbit. The model I have chosen to study is, in a sense, the simplest generalization of the classical studies of the lunar obliquity, adding only the complication of nontrivial inclination and nodal dynamics. For these I use the lunar tidal model of Touma \& Wisdom (1994b). This model is in essential agreement with the tidal model of Goldreich (1966). I find that the dynamical context in which the Moon evolves is considerably more complicated than has been previously assumed. In the phase space there are multiple resonance islands, period-doubled and -tripled islands, and chaotic zones - the whole host of phenomena expected of a nonlinear dynamical system.

I first review the lunar tidal model of Touma \& Wisdom (1994b). I then derive the equations governing the spin dynamics in the model used here. The resulting system is periodically forced and has a single degree of freedom, so it is natural to study the phase space using surfaces of section. I call the resulting Poincaré map the Cassini map. The fixed points of the Cassini map (the periodic orbits) provide a global view, the skeleton, of the dynamics. Surfaces of section for representative epochs along the evolution are displayed. The tidal evolution of the system is discussed, and a new calculation of the tidal heating in the Moon is made. Opportunities for future research are mentioned.

\section{THE LUNAR ORBIT}

Calculation of the history of the lunar orbit is fraught with difficulties. Models of tidal friction are necessarily simplified, and still have many undetermined parameters. Nevertheless, different tidal models give largely the same history of the lunar orbit (Goldreich 1966; Touma \& Wisdom 1994b). These tidal histories exhibit two major problems: the timescale problem and the inclination problem. The timescale problem is that using the current rate of tidal evolution, determined from lunar laser ranging, the Moon is found to have been close to the Earth about 1.5 Gyr ago. The rate of dissipation probably changes as the continents drift, and, evidently, the current rate of tidal dissipation is above average. The inclination problem is that the 
current $5^{\circ}$ inclination of the Moon implies, through the tidal evolution models, that the inclination of the Moon to the equator of the Earth was large when the Moon was close to the Earth. If the Moon was formed by a giant impact followed by reaccretion, one would expect the inclination to the equator to be small. Two potential resolutions of this problem have been proposed (Touma \& Wisdom 1998; Ward \& Canup 2000). Both adjust the inclination when the Moon was close to the Earth.

The tidal evolution model of Goldreich (1966) made quite a few approximations. The orbit of the Moon about the Earth and the orbit of the Earth about the Sun were both assumed to be circular. The equations of motion were multiply averaged over the lunar orbital timescale and the year. The equations for tidal friction, for a number of different tidal models, were similarly averaged. All direct and cross-tidal interactions were included. The model that results still has two timescales, the nodal precession timescale and the tidal evolution timescale. The motion of the node and inclination were determined by integrating the motion on the nodal timescale, and at the same time the tidal equations were averaged on this timescale. The averaged tidal equations were then integrated to get the long-term tidal evolution. The development was noncanonical.

The tidal evolution model of Touma \& Wisdom (1994b) makes the same approximations as Goldreich (1966), but the development is independent and carried out in a canonical framework. A couple of minor errors in Goldreich (1966) were uncovered. The resulting evolution is in good agreement with that calculated earlier. Touma \& Wisdom (1994b) also carried out full unaveraged numerical simulations of the tidal evolution of the Earth-Moon system. That model included the perturbations from all the chaotically evolving planets, the full rigid-body dynamics of the Earth, and all direct and cross-tidal interactions. The integrations were carried out using the symplectic mapping methods of Wisdom \& Holman (1992) and Touma \& Wisdom (1994a). It was found, to our chagrin, that the full numerical integrations were in good agreement with the multiply averaged models. This justifies the use here of the multiply averaged models to represent the orbital evolution of the Moon. Although approximate, it is adequate for a first look at this nonlinear dynamical system.

The history of the eccentricity of the lunar orbit is largely unknown. It is assumed to be zero in the multiply averaged models, and these models are in good agreement with the full numerical integrations starting at the present and integrating backward. But Touma \& Wisdom (1998) considered the forward evolution of the Earth-Moon system, starting with the Moon close to the Earth and near the equator plane. We found that the inclination problem could be resolved if the Moon did a complicated dance through two resonances we dubbed the "evection" and the "eviction." Passage through the evection involved the temporary excitation of large eccentricity. The residual eccentricity could have evolved to larger values later. We suggested that the eccentricity of the Moon might have been large for some early phase of its evolution. In this paper I ignore this possibility and assume zero eccentricity throughout. Presumably, the actual evolution joins the evolutions determined by working backward from the present, at some point unknown. In this paper I am only concerned with the zero eccentricity "standard model." A later work should address the possible effects of orbital eccentricity on the history of the rotation of the Moon.

The details of the multiply averaged model can be found in Touma \& Wisdom (1994b). All tidal models give similar results; here I use only the Mignard tides, with all direct and crosstidal terms. On the nodal timescale the motion of the inclination and the node are exactly periodic, but not uniform. This motion is determined by integrating the averaged equations of motion, with parameters determined by the longer term tidal evolution. This periodic motion of the inclination and node is then used to drive the spin dynamics of the Moon.

\section{SPIN DYNAMICS}

The Moon is assumed to be well described as a rigid body with principal moments of inertia $A<B<C$. The equations of motion are then simply Lagrange's or Euler's equations with gravitational and tidal torques. But this is too general, and some approximations simplify the numerical study (reduce the computational demands), as well as simplify the interpretation.

First, I assume that there is principal-axis rotation. This is a natural consequence of tidal friction in the Moon. But keep in mind that the kinematics of precession require that there be some component of non-principal-axis rotation. Second, I assume that the rotation is always exactly uniform and synchronous. Thus, the small forced libration of the Moon is ignored, but the eccentricity has been set to zero anyway. Third, I include only gravity gradient torques. Dissipation is not included. Finally, I average the equations of motion over the orbital/rotational timescale. These approximations are standard in the treatment of Cassini states (Peale 1969). The new feature here is the use of nontrivial orbital dynamics on the precession timescale.

I make one additional approximation. The synchronous rotation of the Moon with its orbital motion is maintained dynamically by the difference of the moments $A$ and $B$. As others have before me, I am assuming exact synchronous rotation. So there is no need for $A$ to be different from $B$. For this study I set $A=B$. A more precise treatment should include nonzero $(B-A) / C$, but this is not necessary in this first examination of this problem.

The Cassini dynamics is mainly governed by $(C-A / 2-$ $B / 2) / C$, and, to a good first approximation, is independent of $(B-A) / C$ (Peale 1969). Peale (1969) pointed out that $(C-$ $A / 2-B / 2) / C$ for the Moon is hydrostatic for a lunar semimajor axis near $15 R_{\oplus}$. Williams et al. (2001) place the hydrostatic radius near $21 R_{\oplus}$. Other moment differences are hydrostatic at larger radii; all the moments of the Moon are not consistently hydrostatic at any radius. I have set $(C-A) / C=6.3 \times 10^{-4}$ (Yoder 1995) and assume it is hydrostatic inside $15 R_{\oplus}$. I focus this study on the region outside this radius.

I use a Hamiltonian formulation. The dynamical spin state of the Moon is given in terms of Andoyer canonical variables, with respect to an inertial reference. Peale (1969) develops the equations of motion with respect to a system of coordinates that are uniformly precessing with the orbit; this does not make sense here. For a description of the full set of Andoyer variables see Touma \& Wisdom (1994b); I follow their notation here. Since I am assuming principal-axis rotation and that $A=B$, the Hamiltonian for the free rotation (the kinetic energy) is trivially $L^{2} /(2 C)$, where $L$ is the Andoyer canonical momentum that is the component of the total angular momentum along the axis of maximum moment $C$. It is conserved because the conjugate coordinate $l$ does not appear in the full Hamiltonian when $A=B$.

The gravity gradient torque may be derived from the gravitational interaction energy of an out-of-round body (the Moon) with a distant point mass (the Earth),

$$
V=\frac{3}{2} \frac{G M_{\oplus}}{a^{3}} \frac{a^{3}}{r^{3}}\left(\alpha^{2} A+\beta^{2} B+\gamma^{2} C\right),
$$




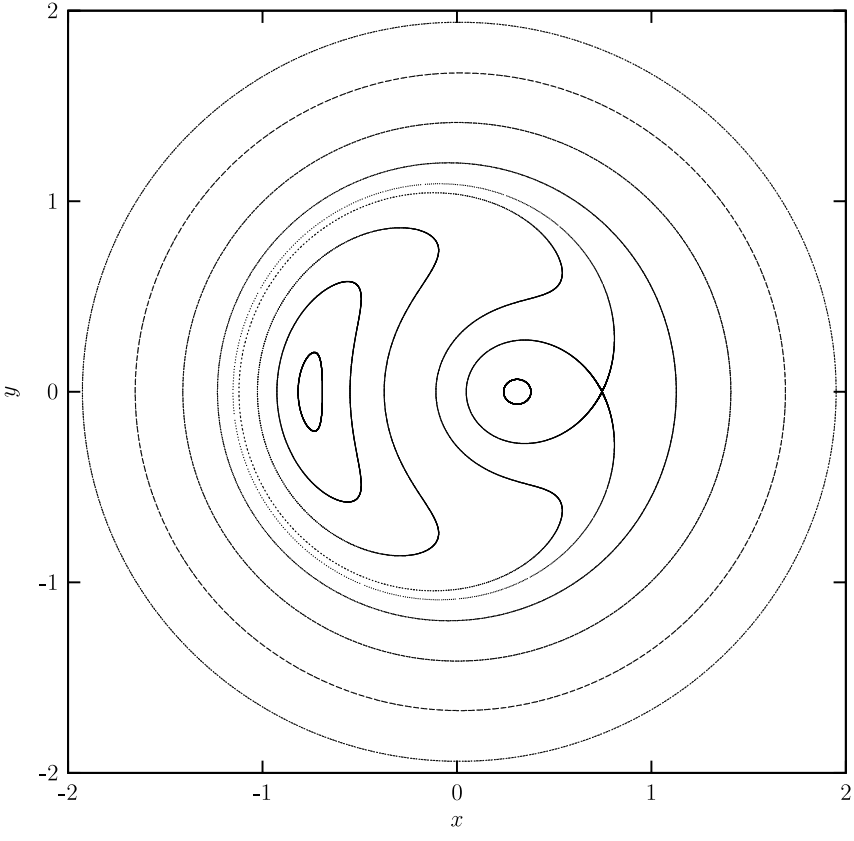

FIG. 1.-Surface of section for the familiar Cassini problem with uniformly regressing node and constant inclination. Here the inclination is $5^{\circ}$, and the period of the node is 1500 orbit periods. The canonical pair $(x, y)$ is plotted when the node of the Moon coincides with the equator of the Earth. Cassini state 2 is the fixed point in the crescent-shaped island. State 1 is the fixed point in the oval island. State 4 is the unstable fixed point at the separatrix crossing.

from Sussman \& Wisdom (2001), where $\alpha, \beta$, and $\gamma$ are the direction cosines of the principal axes $\boldsymbol{a}, \boldsymbol{b}$, and $\boldsymbol{c}$ with respect to the line between the two bodies, $a$ is the semimajor axis, $r$ is the distance between the two bodies, $M_{\oplus}$ is the mass of the Earth, and $G$ is the gravitational constant.

Here I have assumed zero eccentricity, so $a=r$. The factor $G M_{\oplus} / a^{3}$ can be approximated as $n^{2}$, where $n$ is the mean orbital motion of the Moon. Up to constant terms as far as the rotational dynamics is concerned, the interaction energy becomes

$$
V=\frac{3}{2} n^{2}\left[\beta^{2}(B-A)+\gamma^{2}(C-A)\right] .
$$

Here I am assuming $A=B$, so there is a single term.

The Cassini dynamics occurs on the precession timescale, which is much longer than the orbital timescale, so I can safely average over the orbital timescale. The average is straightforward, and the result agrees with the results of Peale (1969) and Ward (1975); the average interaction energy is

$$
V=\frac{3}{4} n^{2}(C-A)\left[1-(\cos \theta)^{2}\right],
$$

where $\theta$ is the obliquity of the spin angular momentum to the lunar orbit normal,

$$
\cos \theta=\sin I \sin i \cos (h-\Omega)+\cos I \cos i,
$$

where $I$ is the obliquity of the spin angular momentum to the normal of the Earth's orbit, which is here the inertial reference plane, $i$ is the inclination of the orbit of the Moon, the Andoyer canonical coordinate $h$ is the ascending node of the spin angular momentum, and $\Omega$ is the ascending node of the orbit of the Moon. The Andoyer momentum conjugate to $h$ is $H=G \cos I$, and $G=L=C n$ is the conserved spin angular momentum ( $G$ and $L$ are canonical Andoyer momenta). The Hamiltonian for the Cassini problem that governs the evolution of the canonical pair $(h, H)$ is just the interaction energy $V$, expressed as I have in terms of the canonical coordinates. The contribution to the kinetic energy from the Andoyer $L$ just implies that the magnitude of the angular momentum is conserved. The time-dependent quantities $i$ and $\Omega$ are determined by the lunar model.

In these Andoyer coordinates, Hamilton's equations have a coordinate singularity for $I=0$, which is in the phase space region of interest. A canonical transformation can be made to remove the singularity. First perform the canonical transformation

$$
\begin{aligned}
H^{\prime} & =C n-H, \\
h^{\prime} & =-h .
\end{aligned}
$$

Note that $\mathrm{Cn}-\mathrm{H}$ is nonnegative. Then perform a second canonical transformation,

$$
\begin{aligned}
& y=\sqrt{2 H^{\prime}} \sin h^{\prime}=2 \sin (I / 2) \sin h^{\prime}, \\
& x=\sqrt{2 H^{\prime}} \cos h^{\prime}=2 \sin (I / 2) \cos h^{\prime},
\end{aligned}
$$

where $y$ is the coordinate conjugate to the momentum $x$. In these variables Hamilton's equations are nonsingular for $I=0$, as desired. They do, however, still have a singularity for $I=\pi$, but this is out of the range of interest in the current investigation (solid body tides are presumed to bring the spin to a prograde, $I<\pi / 2$ configuration). In expressing the equations of motion, it is convenient to use the nonsingular variables for $i$ and $\Omega$ that were introduced in Touma \& Wisdom (1994b). There is no devil in the details; it is just messy, and so will not be further exhibited.

\section{EXPLORATION}

Under the approximations that I have made, the Cassini problem has been reduced to a 1 degree of freedom problem with periodic time dependence. It is therefore natural to study the possible motions of the system using surfaces of section. The

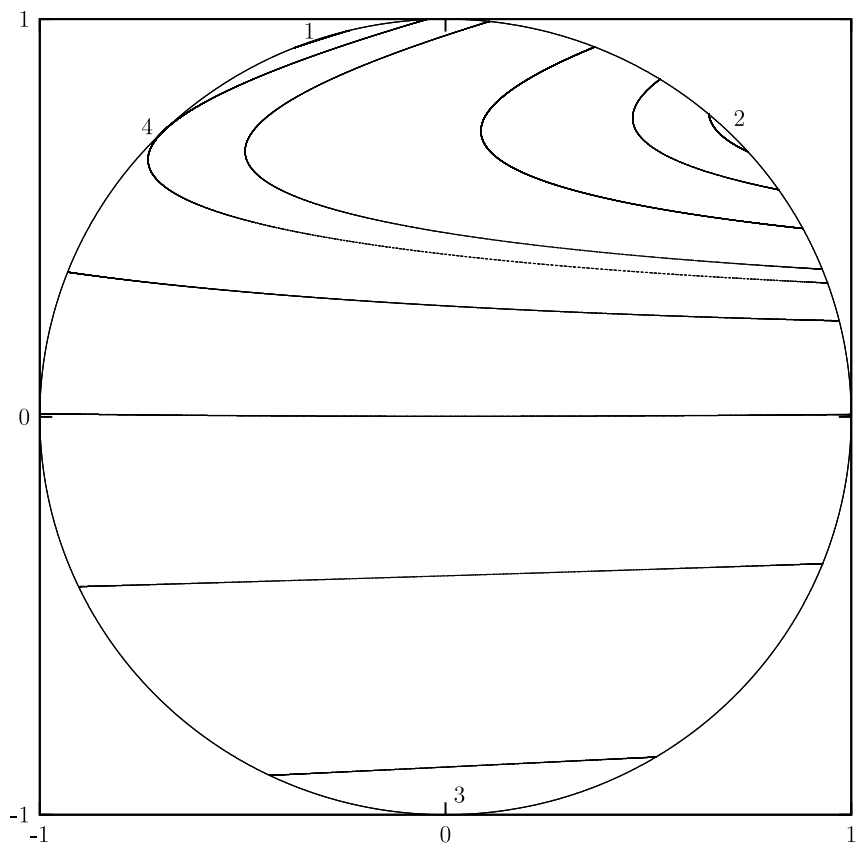

FIG. 2.-Direction of the angular momentum vector projected on the plane perpendicular to the uniformly regressing node of the lunar orbit. 


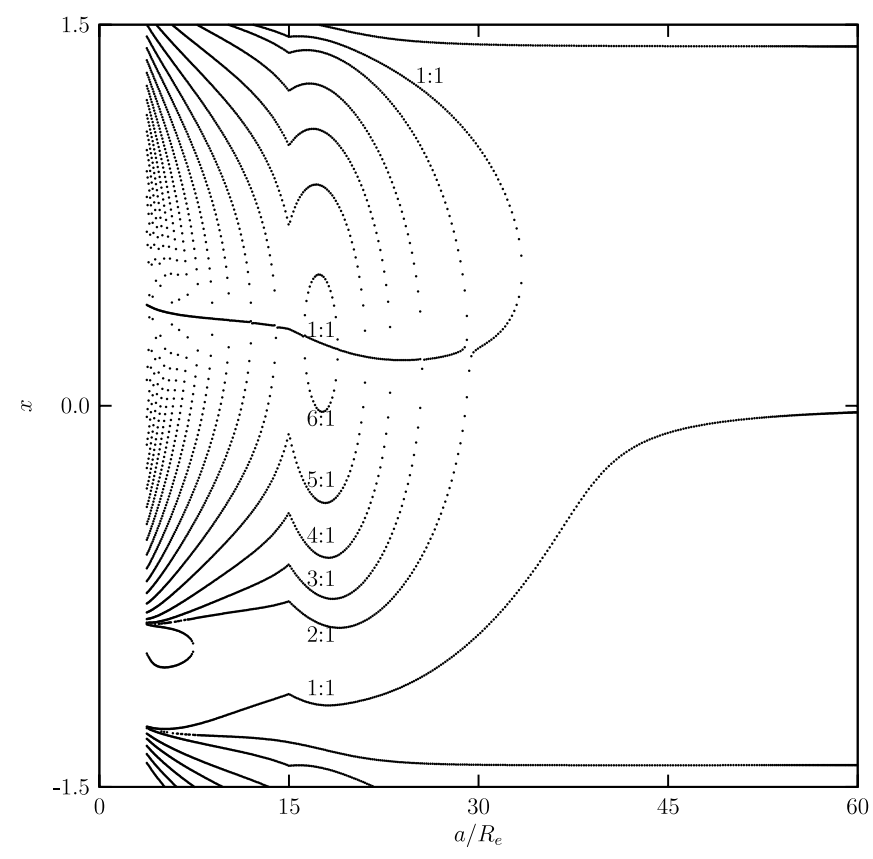

Fig. 3.-Fixed points of the Cassini map on the $x$-axis vs. the semimajor axis of the lunar orbit. The families are labeled by the ratio $j: k$; the spin regresses $j$ times for $k$ nodal regressions.

Cassini map is made by plotting the canonical pair $(x, y)$ whenever the ascending node of the Moon on the ecliptic is the same as the ascending node of the Earth's equator, which is the origin of longitudes.

To set the stage for the exploration of the Cassini map, I first display a section (see Fig. 1) for the familiar Cassini states, i.e., for a uniformly regressing lunar orbit of constant inclination, here $5^{\circ}$. For this section the node regresses with a period 1500 times the lunar orbit period. This model is integrable, since the time dependence can be removed by a canonical transformation to a uniformly rotating frame. For each orbit in the section, I display in Figure 2 the evolution of the direction of the angular momentum on the unit sphere. Here the point is projected on the plane perpendicular to the line of the uniformly regressing node of the orbit. The correspondence between the orbits in the two figures is readily made. There are four points where the projected trajectories are tangent to the unit bounding circle. These are the equilibrium Cassini states. Starting at the top just to the left of center and going around clockwise, Peale (1969) labels these Cassini states 1, 2, 3, and 4. State 4 is dynamically unstable; the others are stable. On the section, Cassini state 2 is the fixed point in the crescent-shaped island, state 4 is the unstable equilibrium, and state 1 is the other stable fixed point in the small circular region. The other stable state 3 is not visible in this section. It is not computed because of the remaining coordinate singularity that I have not bothered to remove. But it would lie near $(x, y)=(-2,0)$. For a faster regression of the node there are only two Cassini equilibria, states 2 and 3 . The section (not shown) has a fixed point near the origin, corresponding to state 2 . This is surrounded by invariant curves. On the angular momentum sphere, state 2 is a fixed point near the north pole. Other orbits just regress around the sphere. Cassini state 3 is a fixed point near the south pole.

Exploring the Cassini map, I find that the largest islands have a fixed point on the $x$-axis. Therefore, a global picture of the possible motion can be obtained by finding all the fixed points on the $x$-axis. I have studied 437 epochs along the tidal evolu-

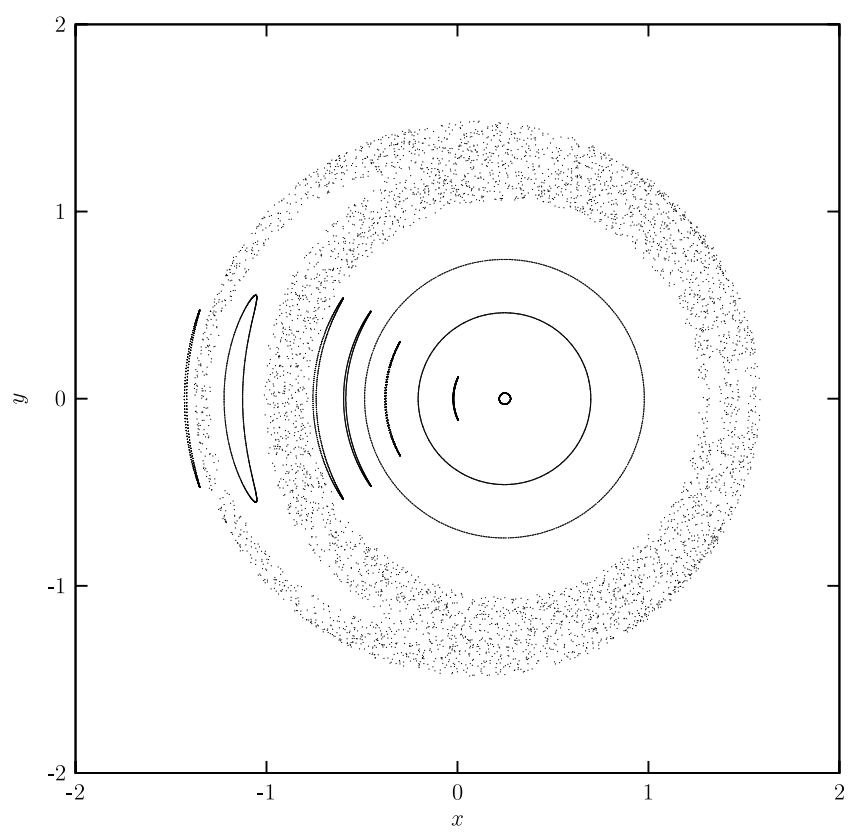

FIG. 4.- Surface of section for $a=17.460 R_{\oplus}$. The canonical pair $(x, y)$ is plotted when the node of the Moon coincides with the equator of the Earth.

tion of the lunar orbit that span the semimajor axis range $3.75-$ $60 R_{\oplus}$. For each state of the tidal system I did a systematic search for fixed points of the Cassini map on the $x$-axis over the interval -1.5 to 1.5 (roughly the part of the phase space with $I<\pi / 2$ ). The results are plotted in Figure 3 . The picture is much more complicated than the constant-precession Cassini model would lead one to expect. That model predicts only one or three fixed points in this part of the phase space, depending on parameters.

Let us now examine a surface of section for a semimajor axis of $17.460 R_{\oplus}$, shown in Figure 4 . A dominant feature of the section is the large chaotic zone. With this are plotted a few representative orbits. Whenever the fixed points of the Cassini map are at the center of a stable island, I have chosen an orbit in that island. There are also a couple of nonresonant orbits. On the diagram offixed points (Fig. 3), the semimajor axis of $17.460 R_{\oplus}$ is near the center of the circle to the left of center of the diagram. There are 16 fixed points plotted. Most of them are unstable. The correspondence between the fixed points and the section is as follows: the fixed point with the smallest $x$ corresponds to the island to the left of the chaotic zone. It is a $-1: 1$ resonance. It is a prograde precession that completes one cycle in a nodal period. I use the notation of $j: k$ to mean the motion is, on average, periodic with $j$ regressions of the spin for $k$ regressions of the node. The next fixed point above this is a $0: 1$ unstable fixed point in the chaotic zone (it does not precess during a nodal period). The fixed point above this is the $1: 1$ resonance, one of the familiar Cassini states (state 2). The spin makes one regression per nodal regression. The fixed point rests in the large crescent-shaped island in the chaotic zone. The next fixed point is a 2:1 unstable fixed point in the chaotic zone. Above this is the fixed point for the $3: 1$ resonance. This is the island just to the right of the chaotic zone. The next fixed point up is the $4: 1$ and corresponds to the second island to the right of the chaotic zone. This is followed by the $5: 1$ and $6: 1$ fixed points, corresponding to the next two islands. Finally, the next fixed point, which is a part of the roughly horizontal line near the middle of the fixed point diagram is a 1:1 fixed point. This is another of the familiar Cassini states (state 1). The fixed points 


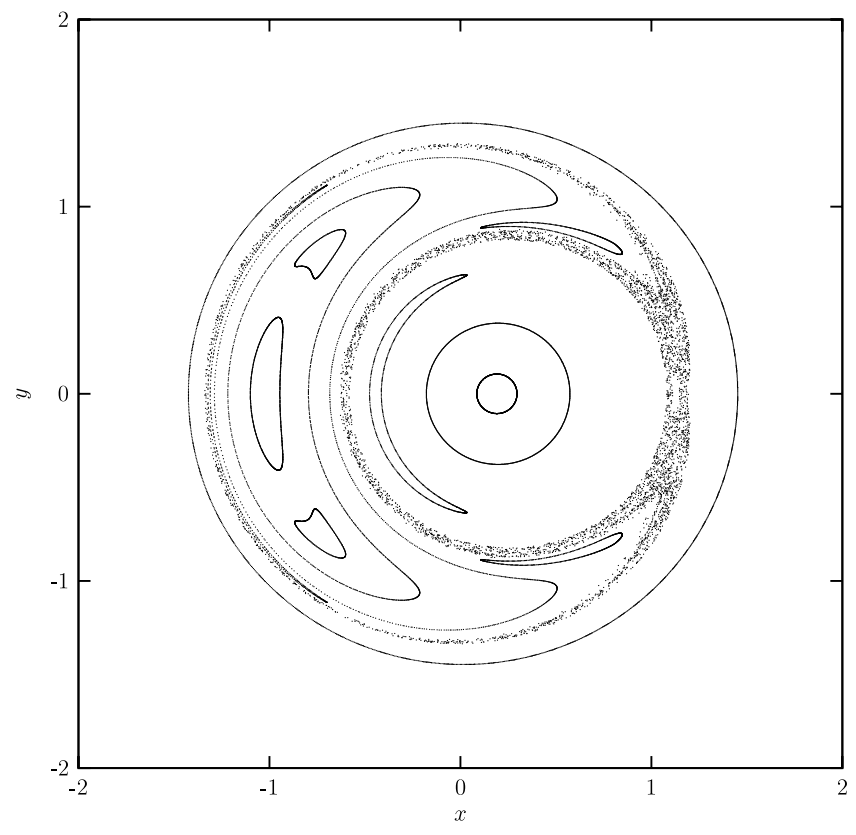

FIG. 5.- Surface of section for $a=26.865 R_{\oplus}$. The canonical pair $(x, y)$ is plotted when the node of the Moon coincides with the equator of the Earth.

above this point are all unstable. They may be paired with the fixed points below the horizontal line. If the one below is stable, then the corresponding one above is the unstable one for the separatrix surrounding the resonance island. What a tour! Of course, much more structure would be seen if the section were examined in greater detail.

Next consider the Cassini map for $a=26.865 R_{\oplus}$, shown in Figure 5. Here the large crescent-shaped 1:1 island shows a period-doubled pair of secondary islands. As the tidal evolution proceeds, this period-doubled pair collapses on the 1:1 fixed point. This occurs for a semimajor axis near $27.4 R_{\oplus}$. The smaller crescent-shaped island belongs to the 2:1 family. There is still a sizable chaotic zone. The teardrop-shaped pair near the chaotic zone actually is part of a triple; the third island is snugly wrapped against the outer edge of the large 1:1 island.

An interesting feature in the diagram of fixed points (Fig. 3) is the break in the $1: 1$ family where it meets the $2: 1$ family. A section for a semimajor axis of $29.081 R_{\oplus}$, near this epoch, is shown in Figure 6. The 1:1 fixed point near the origin undergoes a first-order bifurcation and spawns a pair of $2: 1$ fixed points, one stable and the other unstable.

Several examples of secondary resonances, period-doubled and -tripled islands, have been exhibited. Next I systematically searched for secondary resonances using the same procedure as was used to systematically search for the $1: 1$ fixed points. I looked for period-doubled and -tripled fixed points on the $x$-axis. This time, however, I restricted the search to the interval $-1<x<1$ and $30 R_{\oplus}<a<60 R_{\oplus}$. There are just too many such orbits if the search is extended to smaller semimajor axes. The resulting diagram is shown in Figure 7. It is interesting to see how many low-order secondary resonances there are.

I display two sections that illustrate some families of secondary resonances. First consider the system at a semimajor axis of $32.465 R_{\oplus}$ (see Fig. 8). On this section there is a period-tripled island in the large crescent-shaped region. On the bifurcation diagram (Fig. 7), this is the 1:3 family in the lower left. There is also a period-tripled island in the oval region to the right of center on the section. This corresponds to the $1: 3$ family in the upper left part of the bifurcation diagram. The fixed point that



FIG. 6. - Surface of section for $a=29.081 R_{\oplus}$. The canonical pair $(x, y)$ is plotted when the node of the Moon coincides with the equator of the Earth.

each triple surrounds is the corresponding $1: 1$ family in the bifurcation diagram. Just outside the chaotic separatrix is another period-tripled island. Two islands are on the right, the third is wrapped snugly against the chaotic zone on the left of the section. This is the $1: 3$ family that begins to the left of center in the bifurcation diagram, and arcs to the upper left and lower left. Finally, there is a period-doubled island: one island is on the right and the other is stretched out to the left of the tripled island on the left. It is interesting that the two islands on the left are wrapped so closely to the chaotic zone.

The oval loop in the chaotic zone and the $1: 1$ fixed point in this loop disappear for a semimajor axis greater than approximately $33.4 R_{\oplus}$. On the bifurcation diagram, this region is to the

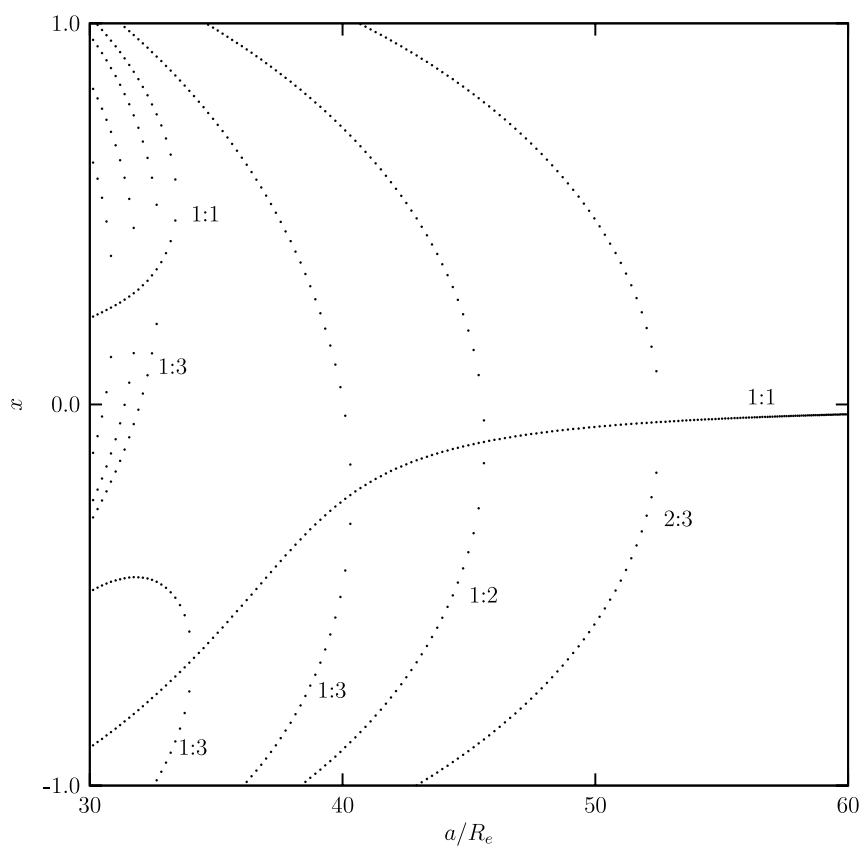

FIG. 7.-Fixed points, including period-doubled and period-tripled, of the Cassini map on the $x$-axis vs. the semimajor axis of the lunar orbit. 


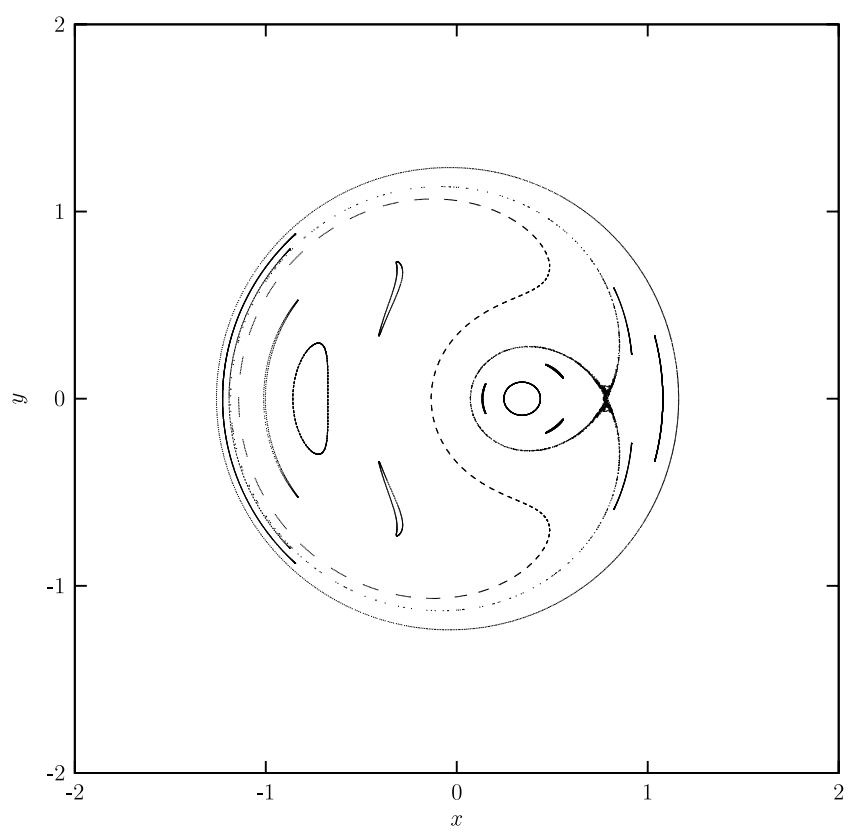

FIG. 8.-Surface of section for $a=32.465 R_{\oplus}$. The canonical pair $(x, y)$ is plotted when the node of the Moon coincides with the equator of the Earth.

right of the 1:1 family in the upper left. The sections are somewhat simpler for semimajor axes greater than $33.4 R_{\oplus}$, but, as is evident in the bifurcation diagram, there are still secondary islands. Figure 9 shows a surface of section for a semimajor axis of $39.912 R_{\oplus}$. The section displays two period-tripled islands and one period-doubled island. The correspondence with the bifurcation diagram is readily made.

\section{TIDAL EVOLUTION}

Ward (1975) argued that early in its evolution, the Moon, through internal friction, would settle on the equilibrium Cassini state with low obliquity (state 1). Examination of the bifurcation diagram (Fig. 3), however, shows that there are many other possibilities. The Moon could have been captured in any of the $n: 1$ islands shown. In addition, there are numerous families of period-doubled and -tripled islands, which are not shown (to reduce the clutter), that could have captured the Moon. But a distinctive feature of the bifurcation diagram is that all these islands eventually collapse on the central 1:1 fixed point (state 1). So the dynamical history may be more complicated than pictured by Ward (1975), but the end result is the same.

After the Moon settles on the 1:1 fixed point, it continues to evolve outward. Where each family of orbits meets the $1: 1 \mathrm{fam}-$ ily there is a bifurcation in the phase space. The most prominent of these bifurcations was illustrated in Figure 6. Examination of the bifurcation diagram shows that permanent capture into these other resonances is not possible, but as each is passed there will be a small hiccup in the obliquity and consequent rate of tidal heating.

In this model, this 1:1 family disappears at approximately $33.4 R_{\oplus}$. The Moon goes through a period of nonequilibrium oscillation as it damps down to another equilibrium. It is probably initially chaotic, but the chaotic zone is apparently quite small (at least in this model), so any chaotic phase probably does not last long. The secondary resonance bifurcation diagram (Fig. 7) shows that the $1: 3$ family around the $1: 1$ in the lower left part of the diagram persists briefly after the upper $1: 1$ family disap-

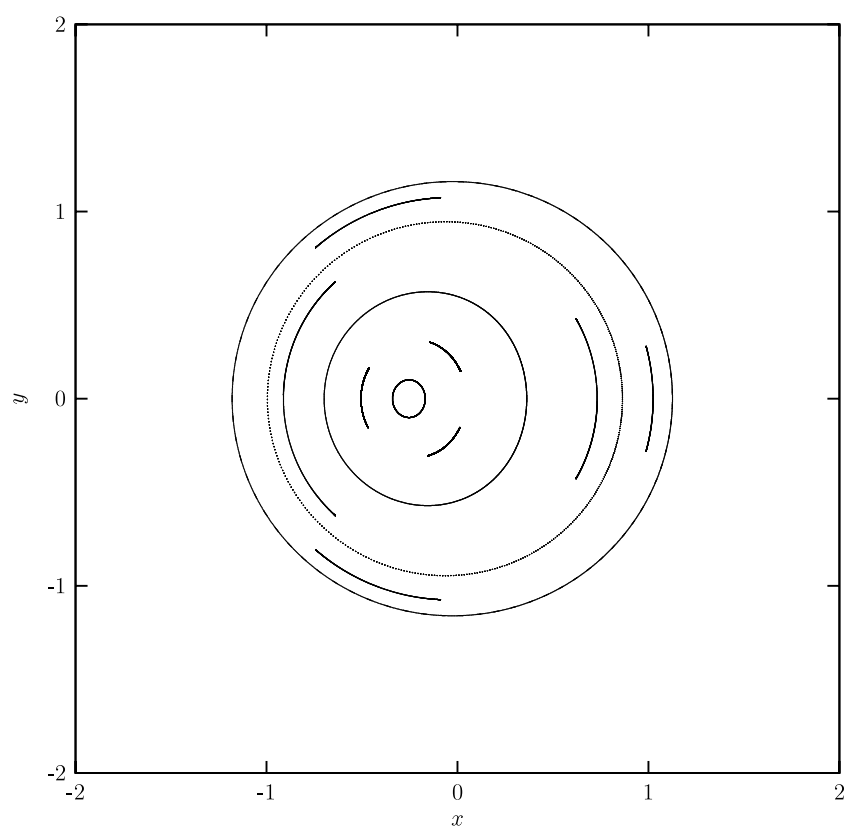

FIG. 9.-Surface of section for $a=39.912 R_{\oplus}$. The canonical pair $(x, y)$ is plotted when the node of the Moon coincides with the equator of the Earth.

pears. If the Moon damps rapidly enough the system could be captured into this $1: 3$ state. But with little further evolution this state also collapses onto the remaining $1: 1$ island with low obliquity. The Moon then remains in this state for its remaining evolution to the present.

The evolution crosses several families of secondary resonances. But the bifurcation diagram shows that these are encountered in the direction that does not allow capture. So, at most, there are other small hiccups in the obliquity and tidal heating rate as each secondary resonance is passed.

\section{A SPECULATION}

There is one speculative dynamical possibility. I noted above that just before the 1:1 family in the upper left part of the bifurcation diagram disappears the chaotic zone for this resonance lies very close to the $1: 3$ and $1: 2$ islands that are wrapped snugly against it (see Fig. 8). One can imagine, but I have not shown, that in a more complete model for the motion of the lunar orbit and spin axis, this chaotic zone is broadened sufficiently to engulf one or both of these chains of secondary islands. If this is the case, then the system might be captured by one of these secondary islands after the 1:1 family disappears and the system moves into the chaotic zone. Or perhaps there are other mechanisms to allow capture by one of these secondary resonances. If a mechanism can be found to allow the Moon to be captured in one of these secondary resonances, then the Moon would be forced to remain in a relatively high obliquity state for an extended interval, until tidal evolution brings the system to the point where these secondary islands collapse on the $1: 1$ fixed point. The extended high-obliquity state would result in larger tidal heating than in the familiar evolution.

\section{TIDAL HEATING}

Peale \& Cassen (1978) examined tidal heating in the Moon with the hope that it could help explain the formation of the lunar maria. They were particularly interested in the Cassini transition, where the Moon switches from Cassini state 1 to 


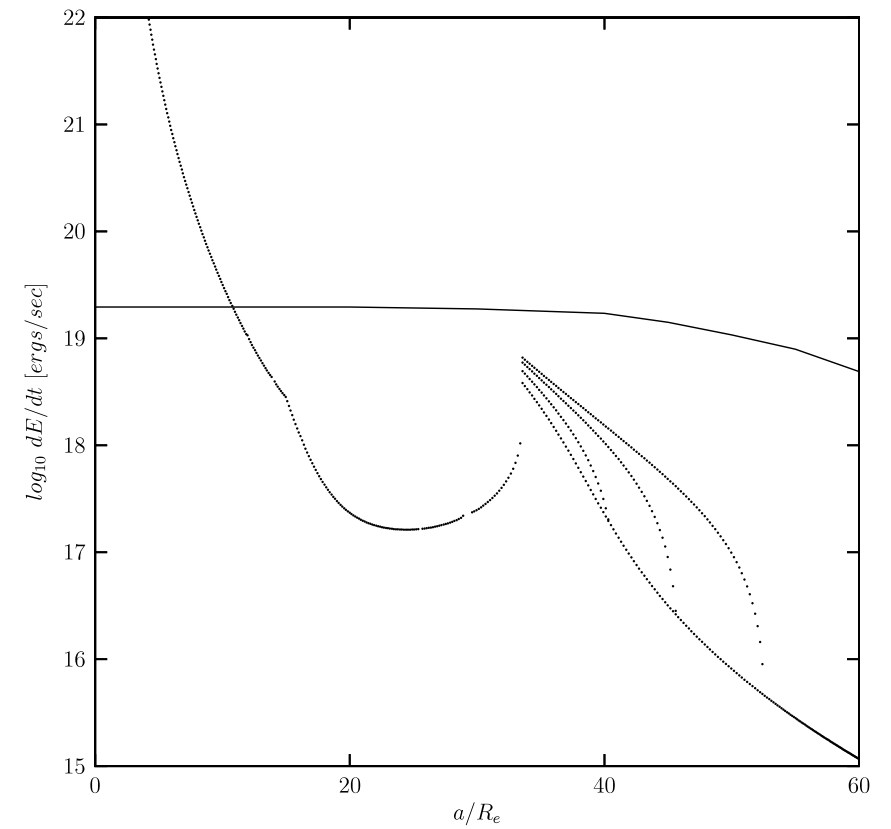

FIG. 10.- Tidal heating rate as a function of the semimajor axis of the lunar orbit. The lower dotted line is for the standard lunar history in which the Moon occupies an equilibrium 1:1 Cassini state. The transition from one equilibrium state to the other occurs at about $33.4 R_{\oplus}$. The three dotted curves above this are for the speculative histories in which the system is captured in one of three secondary resonances. The solid line is the radiogenic heating rate.

state 2 , with a period of high obliquity. They found that although the Moon is more strongly heated during the Cassini transition, this phase of the lunar evolution is so brief that there is no significant consequence for the thermal history of the Moon. In fact, radioactive decay dominates tidal heating throughout most of the evolution. Here I calculate the tidal heating rate for the present model with nonuniform precession and inclination, for both the standard and speculative obliquity histories described above.

The orbit-averaged rate of tidal heating in a uniform satellite with obliquity $\theta$ is (Wisdom 2004)

$$
\frac{d E}{d t}=\frac{3}{2}(\sin \theta)^{2} \frac{3 h_{2}}{5} \frac{1}{Q} \frac{G M_{\oplus}^{2} n R^{5}}{a^{6}}
$$

where $h_{2}$ is the displacement Love number, $Q$ is the effective tidal dissipation parameter, and $R$ is the radius of the satellite. Here I compute the average tidal heating rate in the Moon by averaging this expression over the nodal period. Following Peale $\&$ Cassen (1978) I use $3 h_{2} / 5=0.02$ and $Q=100$. This allows easier comparison with their results, but it is important to note that recent estimates of these parameters (Williams et al. 2001) give several times the heating rate (a factor in the range $3-4)$.

The resulting tidal heating is shown in Figure 10. The heating rate as a function of the semimajor axis is shown. The lower curve is for the standard model in which the system occupies a
1:1 resonance throughout, except for the Cassini transition at about $33.4 R_{\oplus}$. This heating rate is generally consistent with that calculated by Peale \& Cassen (1978), although at small semimajor axes I get a somewhat larger value. This is probably due to the fact that I set the ratio $(C-A) / C$ to the hydrostatic value below a semimajor axis of $15 R_{\oplus}$, whereas Peale \& Cassen (1978) set it to hydrostatic below a semimajor axis of $24 R_{\oplus}$, just after they note that the current value is hydrostatic for $15 R_{\oplus}$. More recent estimates of the distance for which $(C-A) / C$ is hydrostatic are larger, perhaps as large as $23 R_{\oplus}$ (J. G. Williams 2005 , private communication). But the difference in calculated heating rate at small semimajor axis is of little consequence.

The three dotted curves above the standard heating curve give the heating rate for the speculative histories in which the system is captured in one of the secondary resonances. The curves correspond, in order of increasing heating, to the $1: 3$, the $1: 2$, and the 2:3 resonances. The radiogenic heating rate, taken from Peale \& Cassen (1978), is shown as a solid line. The speculative secondary resonance histories, although increasing the rate of tidal heating above the standard model, do not increase the rate above the radiogenic rate. So, alas, they do not need to be considered further.

\section{SUMMARY AND DISCUSSION}

In this paper the dynamics of the lunar spin axis has been examined. Prior work has assumed that the inclination of the lunar orbit is constant and that the node regresses uniformly. This work takes into account the nonconstant inclination and nonuniform regression of the node as determined from averaged models of the motion of the lunar orbit. The resulting dynamics is considerably more rich, exhibiting additional resonances, period doubling and tripling, and chaos.

This study was carried out with the hope that I might find new resonances, probably secondary resonances, in which the Moon might be captured. And, if the state were driven to large obliquity, I might thereby find that tidal heating played a significant role in the formation of the lunar maria. Although I did find that the spin dynamics of the Moon is much richer than had previously been suspected, and I found a significant number of new resonances and secondary resonances, the new resonances are all passed in the direction for which capture does not occur. Thus, they probably play no significant role in the thermal history of the Moon.

I considered a speculative history in which unmodeled effects broaden the observed chaotic zone so that capture into one of the secondary resonances occurs. But even assuming the system is captured in one of these secondary resonances, the tidal heating rate, although increased, is still less than the radiogenic heating. So these speculative histories, even if correct, do not play a significant role in the thermal history of the Moon. The dynamics of the spin axis of the Moon is much richer than previously thought, and can be appreciated for its own sake.

This research was supported in part by NASA through the Planetary Geology and Geophysics program.
Colombo, G. 1966, AJ, 71, 891

Goldreich, P. J. 1966, Rev. Geophys., 4, 411

Laskar, J. 1989, Nature, 338, 237

Laskar, J., \& Robutel, P. 1993, Nature, 361, 608

Peale, S. J. 1969, AJ, 74, 483

\section{REFERENCES}

Peale, S. J., \& Cassen, P. 1978, Icarus, 36, 245

Sussman, G. J., \& Wisdom, J. 1992, Science, 257, 56 MIT)

Touma, J., \& Wisdom, J. 1993, Science, 259, 1294 
Touma, J., \& Wisdom, J. 1994a, AJ, 107, 1189 1994b, AJ, 108, 1943 1998, AJ, 115,1653

Ward, W. R. 1975, Science, 189, 377

Ward, W. R., \& Canup, R. 2000, Nature, 403, 741

Ward, W. R., \& Hamilton, D. 2004, AJ, 128, 2501
Williams, J. G., et al. 2001, J. Geophys. Res., 106, 27933

Wisdom, J. 1985, Icarus, 63, 272

2004, AJ, 128, 484

Wisdom, J., \& Holman, M. 1992, AJ, 104, 2022

Yoder, C. 1995, in Global Earth Physics, ed. T. Ahrens (Washington: AGU), 1 